\title{
Comparison of Different Innovative Technical Solutions for Voltage Regulation in Distribution Grids
}

\author{
P. Franz ${ }^{1}$, I. Talavera ${ }^{2}$, T. Theisen ${ }^{3}$ and J. Hanson ${ }^{4}$ \\ ${ }^{124}$ Department Electrical Power Supply with Integration of Renewable Energies \\ Technische Universität Darmstadt \\ Landgraf-Georg-Straße 4, 64283 Darmstadt \\ Phone/Fax number: +00496151 8645, e-mail: peter.franz@e5.tu-darmstadt.de \\ RWE Deutschland AG \\ Department of New Technologies \\ Kruppstraße 5, 45128 Essen \\ Phone number: T +49 201 12-29387, e-mail: thomas.theisen@rwe.com
}

\begin{abstract}
Due to the increasing amount of Dispersed Generation voltage stability is becoming a more and more critical issue especially in rural distribution grids where most of the generation capacity is installed. Characteristic for these types of grid topologies, which were not designed for a huge share of energy feed-in, are the long lines and the dispersion of consumers. Due to the expansion targets of the German Government regarding renewable energies, it is taken for granted that the voltage problems already appearing in the rural areas will be worsen. In numerous research projects different kinds of technical solutions for improving the voltage deviation have been tested in practice during the last years but often without performing an analytical study of network characteristics. Moreover in most of the projects the impact of only one innovative method was tested but research about the interaction of different technologies is seldom. Thus this paper tries to analyse the benefits of different kinds of innovative equipment and the reciprocal effect of these technologies in four real medium voltage networks in the northwestern part of Germany. Glance at the economic costs of their implementing is also provided.
\end{abstract}

\section{Key words}

Smart grid, voltage regulation, innovative technologies, reactive power support, dispersed generation

\section{Introduction}

The proceeding turnaround in the German electrical power supply, away from nuclear energy generation and towards a renewable energy age, is causing various challenges for the energy sector especially for the existing medium and low voltage networks where most of the Dispersed Power Generators (DPGs) are located. The bulk of this dispersed generation comes from wind power and photovoltaic plants (PV-plants) whose feed-in occurs in areas where the networks were traditionally not designed for the additional function of absorbing generated energy. The dispersed generation causes a bidirectional load flow at the transformers. Thermal overload of grid equipment and voltage problems by exceeding the statuary voltage limits appear more and more often. Regarding the policy roll-out plans of the German Government to increase the share of renewable energies in the gross electricity consumption from $23 \%$ at the end of 2012 to at least $50 \%$ until 2030 (figure 1) it can be taken as granted that these problems will increase [1].

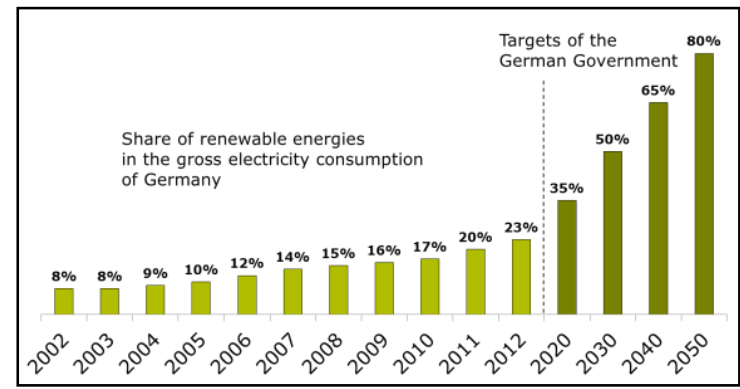

Fig. 1. Share of renewable energies in the gross electricity consumption of Germany [2]

This will cause certainly requirements for grid expansions especially in the low and medium voltage networks where more than $95 \%$ of the $32 \mathrm{GW}$ solar power is already located and most of the further roll-out will take place [3].

Only for these voltage levels a reputable distribution grid study of the German energy agency (dena) foresees grid expansion costs of between 11.4 and 16.2 bn. $€$ depending on the scenario. But the same study also predicts a potential cost reduction by the use of innovative grid equipment like adjustable power transformers, reactive power control, compensation units or voltage regulators of almost $50 \%$ compared to a typical grid expansion [4]. The majority of reasons for grid expansion in the distribution grids are actually caused by voltage problems exceeding the $\pm 10 \%$ voltage band of the EN 50160 and not thermal overloading [5]. Considering the $\pm 10 \% \cdot U_{\mathrm{n}}$-limitation at the end consumers and the direct voltage dependency of the low voltage grid of the medium voltage level, not all of the voltage band can be used by the DPGs located in 
the medium voltage grid (MV-grid). Therefore the German BDEW guideline for the connection of DPGs in medium voltage networks recommends the maximum long-term voltage rise only by these units to $2 \%$ [6].

The following pages treat the question which innovative equipment or combination of different technologies is best suited to reduce the costs of grid expansion and fulfil simultaneously the technical frameworks on the basis of four real MV-grids. The analyses are done quantitative and qualitative by the use of power system simulations.

\section{Initial Situation}

\section{A. Grid modelling}

The four analysed distribution grids are located in North Rhine-Westphalia in the western part of Germany. Three of the grids operate with a nominal voltage of $10 \mathrm{kV}$, the operating voltage of grid " $\mathrm{Z}$ " is $30 \mathrm{kV}$. All municipalities in the analysed distribution grids are eminently rural. That means long overhead lines up to more than $10 \mathrm{~km}$, low electricity consumption and an installed generation capacity which is normally higher than the maximum load of the networks (Table I).

Table I. Characteristic of four MV-grids in Germany

\begin{tabular}{|c|c|c|c|c|c|}
\hline \multicolumn{2}{|c|}{ Grid } & W & $\mathbf{X}$ & $\mathbf{Y}$ & $\mathbf{Z}$ \\
\hline \multirow{4}{*}{ 焉 } & PV & 14,1 & 12,0 & 15,7 & 12,0 \\
\hline & Wind & 5,7 & 8,2 & 2,5 & 6,2 \\
\hline & CHP & 14,0 & 0,8 & 5,0 & 1,4 \\
\hline & Total & 33,8 & 21,0 & 23,1 & 19,5 \\
\hline \multirow{3}{*}{ 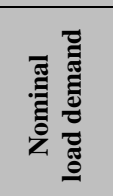 } & $\begin{array}{c}\mathbf{P} \\
(\mathbf{M W})\end{array}$ & 15,4 & 13,6 & 18,5 & 18,5 \\
\hline & $\begin{array}{c}\mathbf{Q} \\
\text { (MVAr) }\end{array}$ & 7,4 & 6,6 & 8,9 & 8,9 \\
\hline & $\begin{array}{c}\text { S } \\
(\mathbf{M V A})\end{array}$ & 17,1 & 15,1 & 20,5 & 20,5 \\
\hline \multicolumn{2}{|c|}{$\begin{array}{c}\text { Ratio } \\
\text { generation/load }\end{array}$} & 2,0 & 1,4 & 1,1 & 1,0 \\
\hline
\end{tabular}

For all consumers where no information about the reactive power demand was available a constant inductive power factor $\cos \varphi$ of 0,95 is assumed and a simultaneity factor of 0,65 is adjusted for all consumers connected directly to the low voltage grid. For consumers with coupling to the medium voltage grid the simultaneity is set to 1 .

For receiving the critical voltage deviations in the grids only the most disadvantageous situations have to be analysed. Therefore two basis scenarios for the worst-case parameter setting of load and generation have been configured: MESMEL and MELZES (Table II).

Table II. Scaling factors for the worst-case scenarios

\begin{tabular}{|l|c|c|}
\hline & MESMEL & MELZES \\
\hline Load & 0,4 & 1 \\
\hline PV & 0,8 & 0 \\
\hline Wind & 1 & 0 \\
\hline CHP & 1 & 0 \\
\hline
\end{tabular}

The "Maximum Energy Supply with Minimum Expected Load" (MESMEL) scenario represents the situation where the highest voltages at the points of common coupling appear. During the "Maximum Expected Load with Zero Energy Supply" (MELZES) scenario the voltages have the lowest values.

The maximum value for the static voltage deviation in the $\mathrm{MV}$-grids is set to $\pm 4 \%$. If the deviation of both scenarios keeps the limitation of $\pm 4 \% \cdot U_{\mathrm{n}}$ at all nodes in the network the frameworks are fulfilled for all other scenarios and there is no need for further simulations. The value of $\pm 4 \%$ is derived from [4] and allows further increase of the voltage deviation along the LV-feeders. The initial results of the load flow without the implementation of any technology solution as well as the ratio of generation and load of the different feeders are shown for grid " $Y$ " in figure 2. The initial reference voltage of the $\mathrm{HV} / \mathrm{MV}$ transformer on the secondary side is set to $1,03 \cdot U_{\mathrm{n}}$. No cable is thermally overloaded, but the maximum voltage deviation comes close to the limit of $\pm 10 \%$ of EN 50160 . The limit of $4 \%$ for the generation units in the MV-grid is already exceeded in several feeders.

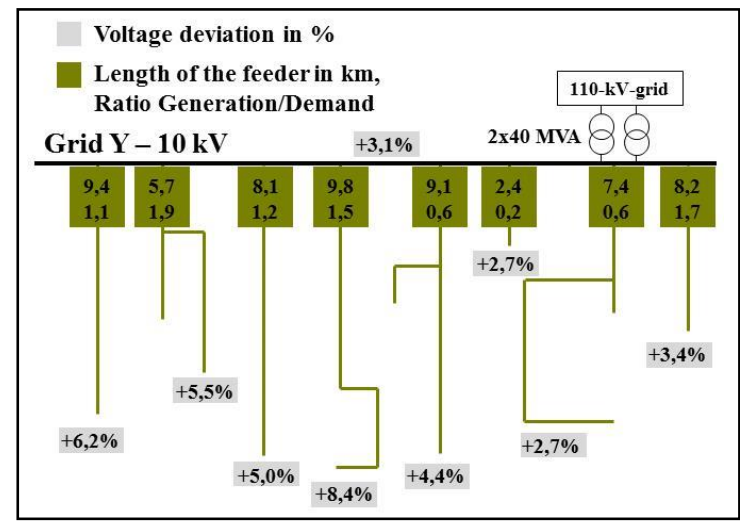

Fig. 2. Max. voltage deviation in grid "Y": No innovative solution included, MESMEL

\section{B. Technical solutions for voltage regulation}

Nowadays there exist numerous possible solutions for voltage regulation in electrical networks. The classical way to solve voltage problems was the strengthening of networks by grid expansion. That means reinforcement by laying new cables with bigger cross-sections or building additional substations for the direct connection of big loads and feed-ins. If the voltage problems are caused by an increase of the demand this method is still the most common solution to reduce voltage drops. However, digging of cable trenches and laying of new cables is with nearly $80.000-140.000 € / \mathrm{km}$ (depending on the degree of urbanization) very expensive and time-consuming [4]. Therefore new innovative solutions for voltage regulation have been tested in several research projects during the last years to limit the voltage deviation and reduce the costs for grid expansion. In most of the projects the impact of only one innovative method has been tested. Beneficial influences of the interaction between different kinds of technologies are seldom, just as the dependency of the effects of the network topology. In Table III an overview of possible solutions for voltage regulation in MV-grids can be found. 
Table III. Technical solutions for voltage regulation [7]

\begin{tabular}{|c|c|c|c|c|c|c|c|c|c|}
\hline Classical & \multicolumn{9}{|c|}{ Innovative Technical Solutions } \\
\hline & \multicolumn{3}{|c|}{ Direct Voltage Regulation } & \multicolumn{2}{|c|}{$\begin{array}{c}\text { Indirect Voltage Regulation } \\
\text { (Reactive Power) }\end{array}$} & \multicolumn{4}{|c|}{$\begin{array}{c}\text { Indirect Voltage Regulation } \\
\text { (Active Power) }\end{array}$} \\
\hline $\begin{array}{c}\text { Grid } \\
\text { Expansion }\end{array}$ & $\begin{array}{l}\text { Wide Area } \\
\text { Control of } \\
\text { HV/MV- } \\
\text { Transformer } \\
\text { (WAVC) }\end{array}$ & $\begin{array}{c}\text { Regulated } \\
\text { Secondary } \\
\text { Distribution } \\
\text { Transformer } \\
\text { (RSDT) }\end{array}$ & $\begin{array}{c}\text { Electronic } \\
\text { Voltage } \\
\text { Regulator } \\
\text { (EVR) }\end{array}$ & $\begin{array}{c}\text { Inverters' } \\
\text { Reactive } \\
\text { Power } \\
\text { Support } \\
\text { (IRPS) }\end{array}$ & $\begin{array}{l}\text { Reactive Power } \\
\text { Compensation } \\
\text { Units } \\
\text { (RPCU) }\end{array}$ & $\begin{array}{c}\text { Energy } \\
\text { Storage } \\
\text { Systems } \\
\text { (ESS) }\end{array}$ & $\begin{array}{l}\text { Active } \\
\text { Demand } \\
\text { Response } \\
\text { (ADR) }\end{array}$ & $\begin{array}{c}\text { Feed-In } \\
\text { Management } \\
\text { (FIM) }\end{array}$ & $\begin{array}{c}\text { Dynamic } \\
\text { Topology } \\
\text { Changes } \\
\text { (DTC) }\end{array}$ \\
\hline
\end{tabular}

Because of different practical reasons and to limit the number of possible technology sets, only the five most promising solutions for voltage regulation in the $\mathrm{MV}$ networks have been selected. The analysis focuses on their technical potential for the reduction of voltage deviation and the costs for grid expansion. The simulations have also been done with combinations of these technical solutions (Table IV). Combinations that are expected to result in little improvement have been excluded.

Table IV. Selected technology sets for voltage regulation

\begin{tabular}{|c|c|c|c|c|c|}
\hline Tech. & WAVC-T & EVR & IRPS & RPCU & ESS \\
\hline Set 1 & $\mathbf{x}$ & & & & \\
\hline Set 2 & & $\mathbf{x}$ & & & \\
\hline Set 3 & $\mathbf{x}$ & $\mathbf{x}$ & & & \\
\hline Set 4 & & & $\mathbf{x}$ & & \\
\hline Set 5 & & & & $\mathbf{x}$ & \\
\hline Set 6 & $\mathbf{x}$ & & $\mathbf{x}$ & & \\
\hline Set 7 & $\mathbf{x}$ & & & $\mathbf{x}$ & \\
\hline Set 8 & & & & & $\mathbf{x}$ \\
\hline Set 9 & $\mathbf{x}$ & & & & $\mathbf{x}$ \\
\hline
\end{tabular}

Wide Area Control of a HV/MV-transformer (WAVC) and the Electronic Voltage Regulator (EVR) belong to the methods based on "direct voltage regulation". That means the voltage is controlled without altering the power flows directly. An indirect increase or decrease of the voltage by modifying the reactive power flow between two nodes by Inverters' Reactive Power Support (IRPS) or the use of Reactive Power Compensation Units (RPCU) is called "indirect voltage regulation via reactive power". The application of Energy Storage Systems (ESS) belongs to the group of "indirect voltage regulation via active power". The use of reactive power to regulate the voltage normally causes higher reactive power flows within the grids and therefore raises the losses in the network. The advantage of voltage control with active power is a higher efficiency in grids with a high R/X-ratio like in MV-grids compared to reactive power regulation.

\section{1) Wide Area Control of HV/MV-Transformer}

The majority of HV/MV-transformers in Germany are equipped with On-Load Tap-Changers (OLTC) to regulate the voltage at the secondary side of the transformer. An OLTC changes the tap of windings without an interruption of supply. For years this has been the only method to regulate the voltage deviation between the high voltage level and the low voltage networks during operation when the demand of the consumers changes. Traditionally these transformers operate with a fixed reference voltage value on the secondary side. The voltage on the secondary side is measured and compared with the reference value. When voltage deviation exceeds a scope of e.g. $1 \%$ the OLTC adjusts the voltage on the secondary side until the final position is reached. This method performs well when the voltage along the lines only deviates in one direction. But this simple principle is not helpful any more in times of high penetration of decentralised generation units with a reversing load flow and thereby reversing voltage profiles. To improve both situations with an OLTC the principle of wide-area control is adopted which means that the voltage at the end of branches in the MV-grids are monitored and used to adapt the reference value for the tap-position.

\section{2) Electronic Voltage Regulator}

In the past Electronic Voltage Regulators (EVRs) were only used by sensitive consumers to protect the equipment against power quality problems. Due to the new appearing voltage deviation problems caused by the dispersed generation, this technology has also found its way into distribution grids. The EVR consists of a voltage source inverter connected in series with a boost injection transformer that injects variable voltage amplitudes on the secondary side. The power electronic unit controls the boost injection of the output. A bypass system is necessary to shunt the boost transformer in case of inverter overloading. As a result the secondary side is directly connected to the primary. This is also the case if the reference voltage on the secondary side equals the voltage on the primary. Because of power electronics the voltage control is smooth and within milliseconds in contrast to the OLTC. The location of an EVR is very flexible. To achieve the requested voltage adaption it is possible to place an EVR within the lines. If the voltage rise along a line is too high the EVR is able to reduce the voltage output between the primary and secondary side without influencing the power factor. The required rating and therefore the cost of the equipment depends on the maximum power flow at the location. Placed in a $\mathrm{MV}$ feeder the EVR can solve the voltage problems of several LV-grids instead of implementing multiple units.

\section{3) Inverters'Reactive Power Support}

The Inverters' Reactive Power Support (IRPS) uses the capability of DPGs to modify their provision of reactive power and thereby influencing the voltage at the point of common coupling. In order to compensate the voltage rise caused by the injected active power of PV-units, inverters create a reactive power flow in the opposite direction by absorbing inductive reactive power. The effectiveness of this method depends on the R/X-ratio of the network. According to the present regulation for DPGs in MV-grids the generators have to be able to provide a power factor of $\cos \varphi=0,95_{\text {underexcited }}$ to $\cos \varphi=0,95_{\text {overexcited [2]. The chosen }}$ parameters for IRPS in the simulations are based on the 
most common $\cos \varphi(P)$-curve of German DSOs (figure 3). The power factor decreases linearly down to 0,95 when more than half of the rated power is supplied.

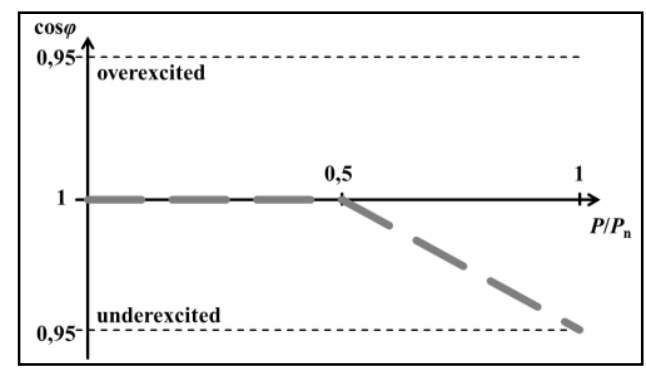

Fig. 3. Common $\cos \varphi(P)$-curve of DPGs in German MV-grids

\section{4) Reactive Power Compensation Units}

In the past Reactive Power Compensation Units (RPCUs) were normally used in the transmission grids to supply the reactive power demand of AC-lines for the transmission of electricity over long distances and in industry with a massive use of asynchronous machines to enhance the power factor. Nowadays RPCUs are also an interesting method in distribution grids for indirect voltage regulation by controlling the reactive power flow. The effects are equivalent to IRPS with the benefit of a direct control of location and amount of reactive power by the DSO. RPCUs can be realized by different equipment. The simplest technologies are mechanically switched reactors and capacitors installed as parallel shunt compensators or series compensators within power lines. The compensators are usually built up out of several single compensation units what allows a stepwise but more or less abrupt control of the reactive power support. To improve the dynamic response and ensure continuous regulation new compensation units with power electronics known as Static VAr Compensator (SVC) can be used. A controller measures voltage and current at the terminal and controls the reactive power support adequately at the node. A SVC design with parallel inductors and capacitors in combination with a small continuous adjustable inductor can reduce the amount of costly power electronics and enable a smooth voltage regulation. Since the effect of reactive power flow is accumulated along the lines, RPCUs are preferably installed at the end of feeders.

\section{5) Energy Storage Systems}

In contrast to the presented voltage regulation methods before, Energy Storage Systems (ESS) can regulate the voltage by changing the load in the feeders and therefore the active power flow. By absorbing energy close to the nodes where it is generated, voltage deviation decreases. A positive effect is the reduction of maximum thermal loading of the equipment what can avoid costs for grid expansion due to overloading. The use of influencing the active power flow for voltage regulation is extremely effective in networks with a relative high $\mathrm{R} / \mathrm{X}$-ratio like it is generally the case in MV- and LV-grids. As the effect of modifying the power flow accumulates along the line, the best position of ESSs for regulating the voltage is at the end of feeders similar to the RPCUs. Whereas the necessary power of the ESS can be easily calculated this is not always possible for the storage capacity especially if we are talking about voltage rises caused by wind power plants. If PV-units are the major reason for voltage deviation, estimations for the range of required energy absorption can be easily done.

\section{Simulation results}

\section{A. Voltage deviation and thermal overload}

For each MV-grid the described technologies (Table IV) have been analysed regarding their capability of reducing voltage deviations. The amount of installed capacity has been chosen in a way to keep the deviation within the limit of $\pm 4 \% \cdot U_{\mathrm{n}}$. Due to the fact that the negative voltage deviation exceeds the bandwidth of $-4 \%$ in only two of almost 30 feeders, the following simulation results are focused on the MESMEL-scenario. Indeed in several of the feeders negative voltage deviation is close to $4 \%$. Thus a reduction of reference voltage at the transformer is not possible and the voltage at the busbar is fixed close to $1,03 \cdot U_{\mathrm{n}}$ for all simulations. Appearing overload problems have been solved by simple grid reinforcements and voltage deviations have been checked again.

By the use of WAVC a reduction of the maximum voltage deviation from approximately $+8,5 \%$ to the $4 \%$-target can be achieved in almost every MV-grid by a regulation of 3-4 tap positions. However, this is not the case for grid "X" where a homogenous improvement of more than $5 \%$ is achieved but still a maximum voltage of $+6 \% \cdot U_{\mathrm{n}}$ remains at the lowest position of the tap-changer (Tab. V). Because of lower voltages with WAVC, power losses increase slightly but no thermal overload appears.

Table V. Max. voltage deviation in grid "X": WAVC, MESMEL

\begin{tabular}{|l|r|r|r|r|r|r|r|}
\hline \multicolumn{1}{|c|}{ in $\%$} & $\begin{array}{c}\text { Feeder } \\
1\end{array}$ & $\begin{array}{c}\text { Feeder } \\
2\end{array}$ & $\begin{array}{c}\text { Feeder } \\
3\end{array}$ & $\begin{array}{c}\text { Feeder } \\
4\end{array}$ & $\begin{array}{c}\text { Feeder } \\
5\end{array}$ & $\begin{array}{c}\text { Feeder } \\
6\end{array}$ & $\begin{array}{c}\text { Feeder } \\
7\end{array}$ \\
\hline Initial & 11,2 & 8,7 & 9,8 & 8,9 & 8,2 & 8,5 & 9,9 \\
\hline WAVC & 4,2 & 4,0 & 4,1 & 4,0 & 4,0 & 4,0 & 4,5 \\
\hline
\end{tabular}

Voltage control with RPCUs is able to limit the maximum deviation to $+4 \%$ in all analysed grids. Figure 4 shows the effectiveness of five RPCUs with an overall rated power of $8 \mathrm{MVAr}$ regulating the voltage in grid "Y". Compared to the initial situation (figure 2) the voltage deviation at the end of the feeders could be reduced from a maximum of $8,4 \%$ down to less than $4 \%$. The absorption of reactive power also causes higher reactive power flows within the lines. These reactive power flows overload $2,1 \mathrm{~km}$ of the line in one feeder.

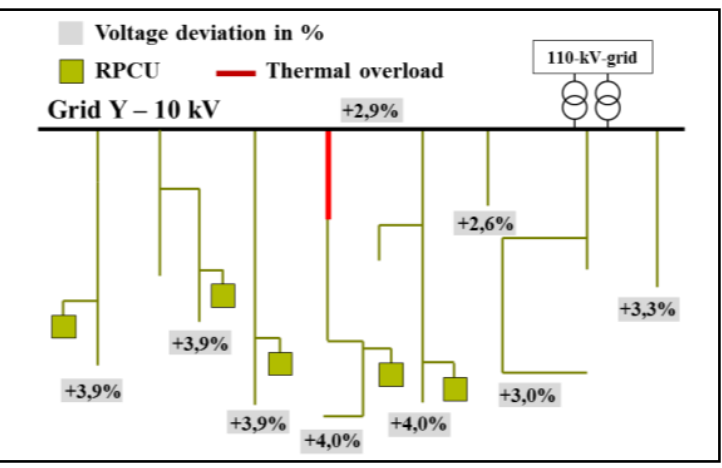

Fig. 4. Max. voltage deviation in grid "Y": RPCUs, MESMEL 
It has to be remarked that the reactive power flow also increases notably the grid losses because of higher loaded cables and HV/MV-transformer.

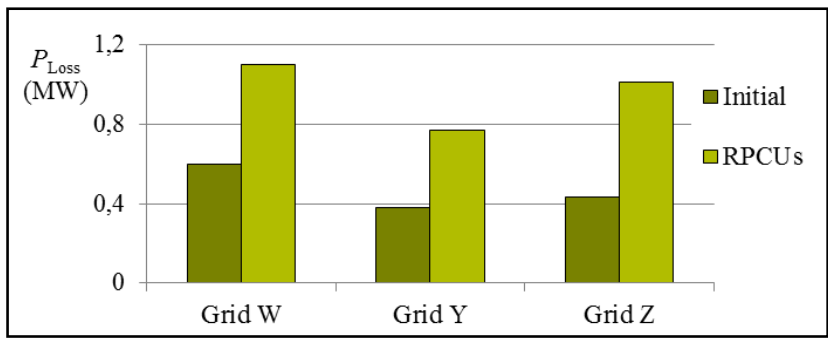

Fig. 5. Active power losses in MV-grids: RPCUs, MESMEL

A voltage limitation of $4 \%$ can also be achieved by the use of EVRs. In grid "Y" four EVRs placed in the feeders where voltage deviation is most critical are needed, each with a rated power of $6 \mathrm{MVA}$. In contrast to RPCUs the power flow is only slightly influenced by lower voltages in the feeders with EVRs. Therefore no thermal overload occurs and the total power losses only slightly rise.

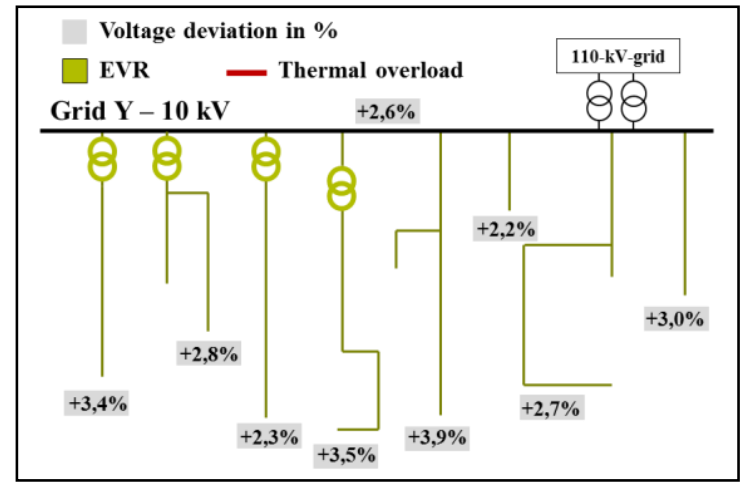

Fig. 6. Max. voltage deviation in grid "Y": EVRs, MESMEL

As it is the case for RPCUs, ESSs are also installed preferably close to the end of lines to boost their effect on voltage. The target of a deviation less than $4 \%$ can be accomplished by around $30 \mathrm{MW}$ of total rated power of the batteries for all four MV-grids (Tab. VI). The batteries only operate with active power compensation. The fundamental advantage of ESSs is the decrease of thermal loading of grid elements and therefore also a reduction of grid losses. This allows the integration of more DPGs without reaching the thermal limits of equipment. However, the expected investment costs for this technology are extremely high.

Table VI. Number and rated power of ESS: MESMEL

\begin{tabular}{|c|c|c|c|c|}
\hline Grid & W & X & Y & Z \\
\hline Number of ESS & 4 & 7 & 7 & 7 \\
\hline Total rated power & $6 \mathrm{MW}$ & $7 \mathrm{MW}$ & $7 \mathrm{MW}$ & $10 \mathrm{MW}$ \\
\hline
\end{tabular}

Regarding the current German guideline the DPGs in MVgrids must be able to operate with a power factor up to 0,95 . The results show that voltage regulation by IRPS has much less effect on the voltage deviation than the methods described before. Due to the location of the DPGs and the relatively high $\mathrm{R} / \mathrm{X}$-ratio of $\mathrm{MV}$-grids, IRPS achieves only small voltage changes (between $1,5 \%$ and $2 \%$ ) and misses the target of $4 \%$ in all analysed grids. Another disadvantage of IRPS is the considerable increase of losses compared to a small reduction of voltage deviation.

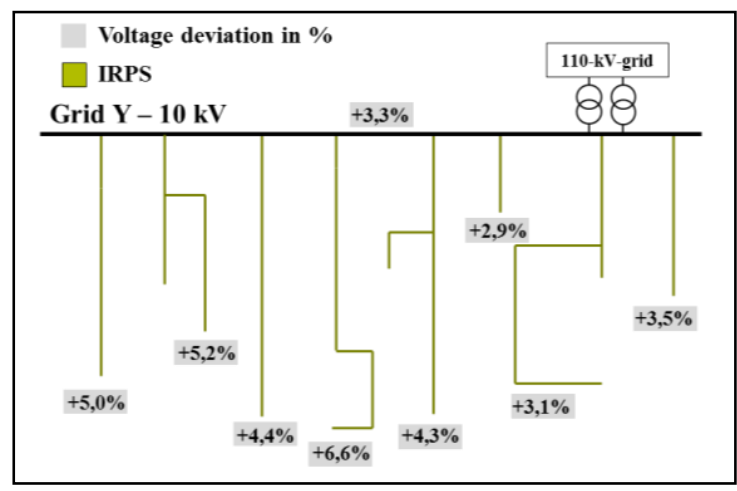

Fig. 7. Max. voltage deviation in grid "Y": IRPS, MESMEL

Nevertheless IRPS should not be lost out of sight as an additive method for voltage regulation, because the use of inverters' ability for regulating reactive power is extremely cheap since no additional measurement or equipment has to be installed in the grid.

\section{B. Comparison of technology sets}

WAVC has figured out to be a very effective method to reduce voltage deviations for a wide range of demand/ generation-combinations. But as you can see for grid " $X$ " there are possible situations where WAVC is not able to reduce voltage deviation within the defined limits if the method is used exclusively. This fact appears in "inhomogeneous" networks consisting of feeders with a complete different ratio of demand and generation. Possible technical solutions are the implementation of technology sets which enlarge the degree of freedom like combinations of WAVC with EVR, IRPS, RPCU or ESS. Particularly WAVC combined with a $\cos \varphi$-regulation of DPGs has turned out to be an effective technology set to achieve further reduction of maximum voltage deviation even in inhomogeneous grids accompanied by the positive effect that no additional costs occur (figure 8, grid " $X$ "). But also combinations with EVRs, RPCUs or ESSs are conceivable since additional equipment is only necessary in critical branches.

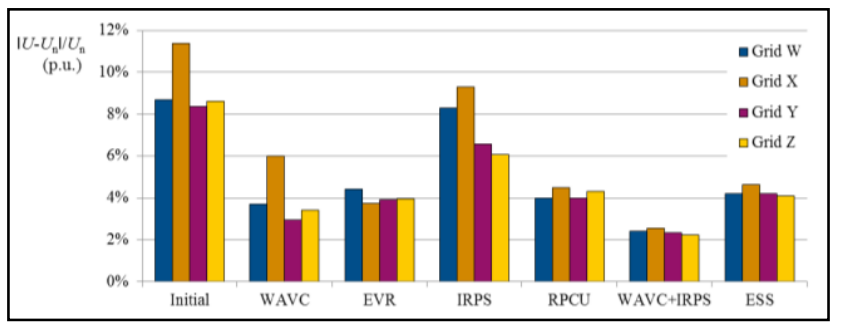

Fig. 8. Comparison of different technology sets: Maximum voltage deviation in MV-grids, MESMEL

When selecting an appropriate technology set, also effects on thermal loading of grid elements have to be considered. Regulation methods using reactive power for an indirect voltage control cause the highest amount of additional loading. If RPCUs are deployed the number of overloaded kilometres surges extremely compared to other methods. But also a combination of WAVC and IRPS increases the number of overloaded lines albeit at a lower level (fig. 9). 


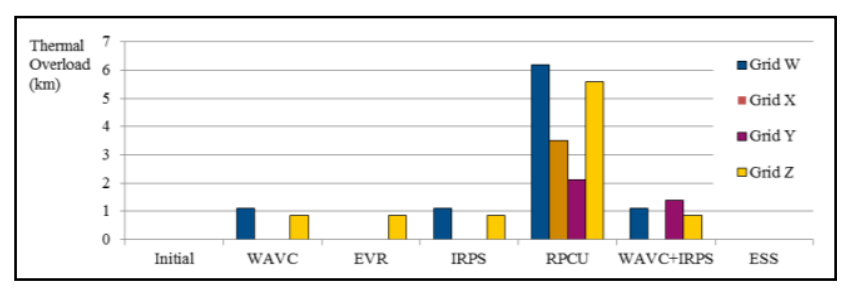

Fig. 9. Comparison of different technology sets: Overloaded lines in MV-grids, MESMEL

\section{Economic outlook}

To get a complete overview of adequate technologies for voltage control also economic aspects must be considered. Looking at the investment costs IRPS and WAVC seem to be the most economical solutions for voltage regulation in MV-grids. Compared to WAVC the costs for EVRs are higher but an EVR can also be an interesting investment for inhomogeneous grids where WAVC is not sufficient any more or if the voltage limit is only exceeded in one of several feeders. If price for power electronics continues to decrease, EVRs could be more economical in the future. Although RPCUs have shown to be a good solution for reducing voltage deviations, from the economic sight of view other technical solutions seem to be advantageous. At the moment the price for power electronics make these units still very expensive and like all methods based on indirect voltage control via reactive power the additional active power losses have also to be taken by DSOs. The use of IRPS causes no significant investment costs. But there are the same two drawbacks for IRPS like for RPCUs even though more moderate: additional operational costs for the DSOs by the additional reactive power flow and possible grid expansions because of thermal overloading of equipment and cables (figure 10).

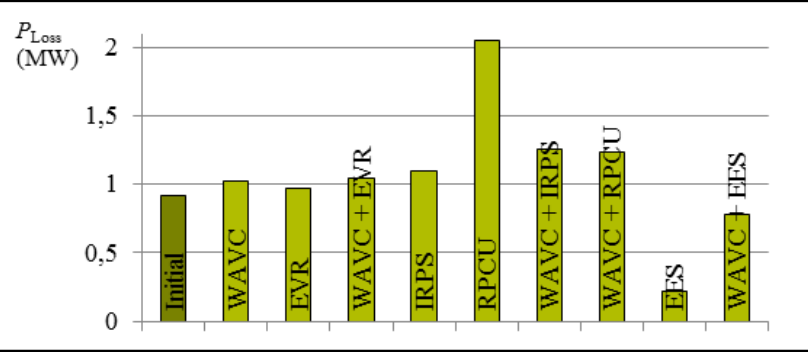

Fig. 10. Comparison of different technology sets: Power losses in grid " $X$ ", MESMEL

ESSs can successfully limit voltage deviations and decrease loading and grid losses simultaneously. However, the required power and storage capacity causes investment costs more than $10 \mathrm{x}$ higher than for other methods. In combination with WAVC the required capacities could be noticeable lower but the expected investments are still very high.

To quantify the costs of an innovative technology for voltage regulation further studies have to be done. Besides investment costs, operational costs, economic lifetime of the equipment and investments in grid expansion have to be considered in order to do a comprehensive economic analysis. Furthermore solutions like RPCU or ESS could generate revenues if they are also used to offer ancillary services. The revenues also have to be taken into account.

\section{Conclusion}

There are at least nine different innovative technology sets to control voltage deviations in distribution grids. WAVC has figured out to be an effective method in the MV-grids. In most of the analysed grids this method is able to limit the deviation below $\pm 4 \% \cdot U_{\mathrm{n}}$. Furthermore additional grid losses and the raise of thermal loading are manageable. Only in grids with feeders with a wide range of ratios of generation and demand problems remain. RPCUs are able to reduce the maximum voltages below the limit of $4 \%$ in all grids but only accompanied by overload and a dramatic increase of grid losses. EVRs can achieve comparable voltage reductions with the advantage of lower grid losses and only moderate rise of the loading factors. A negative aspect is the high number of units for all the feeders where excessive voltage deviations appear. ESSs are also able to achieve the desired decrease of voltage deviations without causing any thermal overload. However, the required capacities are high and the investment costs are far higher than for other technologies. A single use of IRPS only reduces voltage deviation between $1,5 \%$ and $2 \%$ besides accompanied by a notable increase of grid losses. For these reasons innovative methods with a combination of different voltage regulation technologies have shown to be a good solution particular in inhomogeneous networks where possible synergies can be realized. Regarding the expected costs, especially a simultaneously use of WAVC and IRPS seems to be worthwhile. But also combinations of WAVC with EVRs, RPCUs or ESSs could decrease the voltage deviation below the limits in all analysed grids and help to reduce accumulated investment costs. Further studies will be necessary to quantify the total amount of costs consisting out of investment cost, operational cost and additional losses over the lifetime for the different methods of voltage regulation.

\section{References}

[1] Federal Ministry of Economics and Technology (BMWi), First Monitoring Report Energy of the future, Berlin (2012).

[2] Bundesverband der Energie- und Wasserwirtschaft e.V. (BDEW), Erneuerbare Energien und das EEG: Zahlen, Fakten, Grafiken, Berlin (2013).

[3] 50Hertz Transmission GmbH, Amprion GmbH, TenneT TSO $\mathrm{GmbH}$, TransnetBW GmbH, EEG-Anlagenstammdaten zum 31.12.2012, www.eeg-kwk.net, (2012).

[4] Deutsche Energie-Agentur GmbH (dena), Ausbau- und Innovationsbedarf der Stromverteilnetze in Deutschland bis 2030, Berlin (2012).

[5] EN 50160, "Voltage characteristics of electricity supplied by public distribution networks," (2011).

[6] Bundesverband der Energie- und Wasserwirtschaft e.V. (BDEW), Technische Richtlinie Erzeugungsanlagen am Mittelspannungsnetz - Richtlinie für den Anschluss und Parallelbetrieb von Erzeugungsanlagen am Mittelspannungsnetz, Berlin (2008).

[7] B. Gwisdorf, T. Borchard, T. Hammerschmidt, C. Rehtanz, Technical and economic evaluation of voltage regulation strategies for distribution grids with a high amount of fluctuating dispersed generation units, IEEE, (2010). 